\title{
Estado de los bosques de Araucaria angustifolia: especie nativa en peligro crítico
}

State of the Araucaria angustifolia forests: native species in critical danger

\author{
Claudia V. Luna ${ }^{1}$ y María L. Fontana ${ }^{1,2}$ \\ ${ }^{1}$ Cátedra de Silvicultura. Facultad de Ciencias Agrarias- Universidad Nacional del \\ Nordeste. Corrientes, Argentina. Tel.: ++54 3794427589 Int.: 156. Sgto. Cabral 2131- \\ W3402BKG, Corrientes. ARGENTINA. E-mail: claudiaverluna@gmail.com.
Estación Experimental Agropecuaria INTA Sáenz Peña. Presidencia Roque Sáenz Peña, Chaco. Argentina.

Luna, C. V. \& Fontana, M. L. (2017). Estado de los bosques de Araucaria angustifolia: especie nativa en peligro crítico. Revista Estudios Ambientales, 5(2), 79-93.

Recibido: 19 de octubre de 2017

Aceptado: 21 de noviembre de 2017

Publicado: 31 de diciembre de 2017 


\section{RESUMEN}

La familia Araucariaceae está representada en Argentina por el género Araucaria. Araucaria angustifolia es una especie que a pesar de poseer superioridad en cuanto a magnitud respecto a otros árboles con los que compite; por su estado de conservación es considerada en peligro crítico de extinción. En este trabajo se expone de qué manera esta especie ha sido explotada por su madera y la expansión agrícola. Es por ello que este artículo describe el estado del arte de este recurso haciendo énfasis en la importancia desde el punto de vista maderable como así también su utilidad, estado de conservación del recurso y recursos genéticos disponibles, ya que solo a través del conocimiento del patrimonio que poseemos es posible implementar acciones para su conservación, protección e integración de las comunidades locales en dichas actividades.

Palabras clave: Araucariaceae, conservación, recursos genéticos, vulnerabilidad.

\section{ABSTRACT}

The Araucariaceae family in Argentina is represented by the genus Araucaria. Araucaria angustifolia is a species despite having superiority in magnitude over other trees with which it competes; his condition is considered critically endangered. In the context of this work we propose how this species has been exploited for its timber and agricultural expansion. That is why this paper describes the state of art of this resource emphasizing the importance from the point of view timber as well as its utility, state resource conservation and genetic resources, since only through knowledge of heritage we possess is possible to implement actions for conservation, protection and integration of local communities in such activities.

Key words: Araucariaceae, conservation, genetic resources, vulnerability. 


\section{INTRODUCCIÓN}

La familia Araucariaceae es un grupo ampliamente distribuido en el pasado en Europa; actualmente está confinado al hemisferio austral, con taxones muy apreciados desde el punto de vista maderable y ornamental (Guillot Ortiz et al., 2006). Actualmente existen 2 géneros vivientes, Agathis con 20 especies en Australasia y Araucaria con 16 especies, 2 de ellas en Sudamérica (Waters, 2003).

Desde su origen en el Triásico, esta familia amplió su distribución y se ha diversificado en los dos hemisferios en los periodos Jurásico y Cretácico (Herbst et al., 2007). Existe abundancia de registros fósiles para ella, encontrándose hojas, conos y polen desde el Triásico; y de maderas durante el Cretácico, periodo donde se encuentran representadas cerca del $70 \%$ del total de las especies de Sudamérica y Antártida. Material fósil hallado en la Formación Bajo Barreal (Chubut y Santa Cruz en la Patagonia, Argentina), presenta afinidad anatómica no solo con la familia sino también con las especies actuales de Sudamérica, como lo son Araucaria angustifolia y $A$. araucana, sugiriendo que las Araucariáceas habrían dominado el componente arbóreo en la Patagonia durante el periodo Jurásico y Cretácico constituyendo bosques mono específicos (Pujana et al., 2007; Gnaedinger y Herbst, 2009).

Araucaria angustifolia es nativa del sur de Brasil y el norte de Argentina, donde llegó a formar bosques muy extensos; hacia 1960 se contabilizaban unas 200.000 ha como manchones de bosques silvestres en las sierras del norte de Misiones (Haene y Aparicio, 2014). Estos bosques han sido talados con el fin de aprovechar la madera de los árboles y de abrir los terrenos para la agricultura. Luego de siglos de explotación, los bosques de araucarias han quedado reducidos a sólo una mínima fracción de su extensión original y actualmente su estado de conservación es "En Peligro Crítico (CR)" según la UICN (2006).

La recuperación de este ecosistema es urgente y solo puede lograrse con su restauración ecológica; rehabilitando aquellos ambientes degradados y restableciéndolos (Meffey Carroll, 1994). Para lograrlo, es necesario conocer las características ecológicas y los aspectos más relevantes sobre la conservación y hábitat de esta especie tan emblemática en Argentina. Por ello este artículo describe el estado del recurso haciendo énfasis en su importancia económica, utilidad, estado de conservación y recursos genéticos disponibles ya que solo a través del conocimiento 
del patrimonio que poseemos es posible implementar acciones para su conservación, protección e integración de las comunidades locales en dichas actividades.

\section{Género Araucaria Jussieu}

Etimológicamente Araucaria, proviene de la región de Arauco (Indios de Arauco del centro y sudoeste de Chile), en donde se descubrió la primera especie (Guillot Ortiz et al., 2006; Véliz Pérez et al., 2007).

Araucaria angustifolia (Bertol.) Kuntze 1898.

Sinonimia: Colymbea angustifolia Bertol.; Araucaria brasiliana Richard; Araucaria brasiliensis London; Pinus dioica Vellozo.

Nombres comunes: Araucaria Brasileña, Pino del Paraná, Pino de Misiones, Árbol candelabro, Pinheiro-do-paraná, Pinheiro-brasileiro, Curý (FAO, 1973); Cori, Pinheiro caiova, Pinheiro macaco, Pinheiro branco, Kuriý (Salazar et al., 2000).

\section{Distribución geográfica}

Se halla distribuida al noreste de la Argentina, el este de Paraguay y sureste de Brasil, en la región Bosque de la Selva Paranaense en el Bosque Atlántico del Alto Paraná. En Brasil, al sur del trópico de Capricornio, entre altitudes comprendidas entre 50 y 1800 m.s.n.m. También existen poblaciones fragmentadas de $A$. angustifolia en Argentina nororiental y Brasil sudoriental (Silveira Wrege et al., 2009). En Paraguay se encontraron unos pocos ejemplares nativos, los que fueron protegidos creándose una Reserva Nacional a partir de 1971 en Pinalito (departamento de Alto Paraná). En Argentina, se encuentra en el ecotono de las Selvas Mixtas con los bosques de Araucaria de Brasil, donde todas las características de los pinares de Araucaria de Misiones hacen que sean tan representativos como los del área del sur de Brasil (Rodríguez et al., 2005). 


\section{Hábitat y comunidades}

Su comportamiento ecológico le permite prosperar en una amplia variedad de suelos, desde pobres - derivados de la arenisca - hasta ricos provenientes de basalto; que van de profundos a poco profundos, húmedos y adecuadamente drenados.

En la Selva Misionera crece en suelos lateríticos, profundos y permeables, con un $\mathrm{pH}$ ácido. Se encuentra en alturas de entre 300 a 2000 m.s.n.m, con precipitaciones anuales entre 1250 a $2450 \mathrm{~mm}$. Es de clima templado con temperaturas entre 10 y 21 ${ }^{\circ} \mathrm{C}$, y con heladas que ocurren de 10 a 25 días al año (Laharrague, 2003).

Las bajas temperaturas de las elevadas altitudes donde se encuentra distribuido, le permiten manifestar una tolerancia y adaptabilidad que le confiere ventajas respecto a otros árboles con los que compite; es ésta una de las principales características medioambientales que determinan su distribución y por ende su susceptibilidad al cambio climático.

Es una especie muy longeva que llega a vivir unos 400 años con de 35 a 60 m de altura y 0,8 a 2 m de diámetro (Silveira Wrege et al., 2009).

\section{Importancia económica}

Tiene un gran valor maderero; de fácil trabajabilidad, se utiliza para la construcción, cajonería, muebles, laminados, placas de revestimiento, listones, vigas, lápices, carpintería en general, cerillas, postes y mástiles de los barcos. Produce también celulosa de fibra larga y papel de calidad excepcionalmente elevada. Las semillas son una fuente de proteínas para los seres humanos y para la nutrición del ganado doméstico y silvestre. También son útiles los brotes y protuberancias (nudos en el tronco del árbol) empleados en la medicina popular y las artesanías (Bittencourt et al., 2004; Wendling et al., 2009).

Se encuentra en plantaciones forestales comerciales, en los últimos años su demanda presenta un sostenido crecimiento, lo cual ha mejorado los precios en relación con ciclos anteriores; actualmente es mejor paga la madera de araucaria que la de pinos resinosos (Colegio de Ingenieros Forestales de Misiones, 2014).

Además, por la demanda insatisfecha en el mercado, no existen problemas para colocar la producción ya que presenta atributos que la destacan como tener una madera más dura, más resistente y de características estéticas más apreciadas. Hay 
que señalar, que prácticamente toda la madera de araucarias misioneras se destina a aserraderos, donde se le realizan distintos procesos de transformación y agregados de valor (INTA, 2014a).

\section{Estado de conservación del recurso}

El área ocupada por estos bosques a comienzos del siglo $X X$ se ha visto reducida en más del 97\%; debido a la tala indiscriminada y habilitación de terrenos agrícolas (Sebbenn et al., 2003).

Algunos de los agentes que agravan su estado de conservación crítico, se deben al cambio climático; considerándose como factores estresantes principales al déficit hídrico y térmico; además de la erosión genética causada por la presión de selección, dado que se eliminaban los mejores ejemplares adultos y quedaban en pie aquellos generalmente enfermos y con escaso valor para la futura regeneración, con el consiguiente empobrecimiento del 'pool' génico de las poblaciones remanentes (Rodríguez et al., 2005). El ciclo de vida de esta especie es comparativamente más largo que el de otras coníferas y su proceso de adaptación a condiciones ambientales cambiantes más lento que el ritmo pronosticado del cambio climático mundial (Bittencourt et al., 2004; Silveira Wrege et al., 2009).

Ha sido incluida en la Lista Roja de la UICN (Farjon, 2006) en la categoría de "En Peligro Crítico de extinción", a la fecha quedan menos de mil hectáreas en unas pocas áreas naturales protegidas (San Antonio, Cruce Caballero y La Araucaria; en Misiones) (Haene y Aparicio, 2014).

En Argentina, fue sometida a una tala excesiva en los últimos años, al punto que en la actualidad no quedan ejemplares dignos de aprovechamiento industrial. No se cuenta con datos actuales de existencias, Braier (2004) cita una superficie aproximada de 28.500 ha la con ejemplares naturales en sobremadurez o decrepitud que no los hace aprovechables; hecho que demuestra lo comprometida que está la situación de los bosques de Araucaria (Rodríguez et al., 2005). Actualmente Misiones conserva la mayor superficie de selva de serranía de araucarias (Fig. 1); pero cabe aclarar que este remanente no está integrado por rodales continuos, por lo que la superficie efectiva y funcional de este ambiente es menor. En un intento urgente para proteger los últimos remanentes del bosque de pino paraná y su fauna, Misiones declaró a la $A$. 
angustifolia "Monumento Natural" en 1986, prohibiendo su tala mediante ley provincial; una década después, a través de un convenio entre el Ministerio de Ecología y Recursos Naturales Renovables (ME y RNR), la Facultad de Ciencias Forestales de Eldorado y el Centro de Investigaciones Forestales (CIEF), en torno a un proyecto de conservación de recursos genéticos "in situ" y "ex situ" de A. angustifolia, se pretendió garantizar la provisión de semillas de alta calidad genética para las forestaciones comerciales de la provincia (Fundación Azara, 2009).

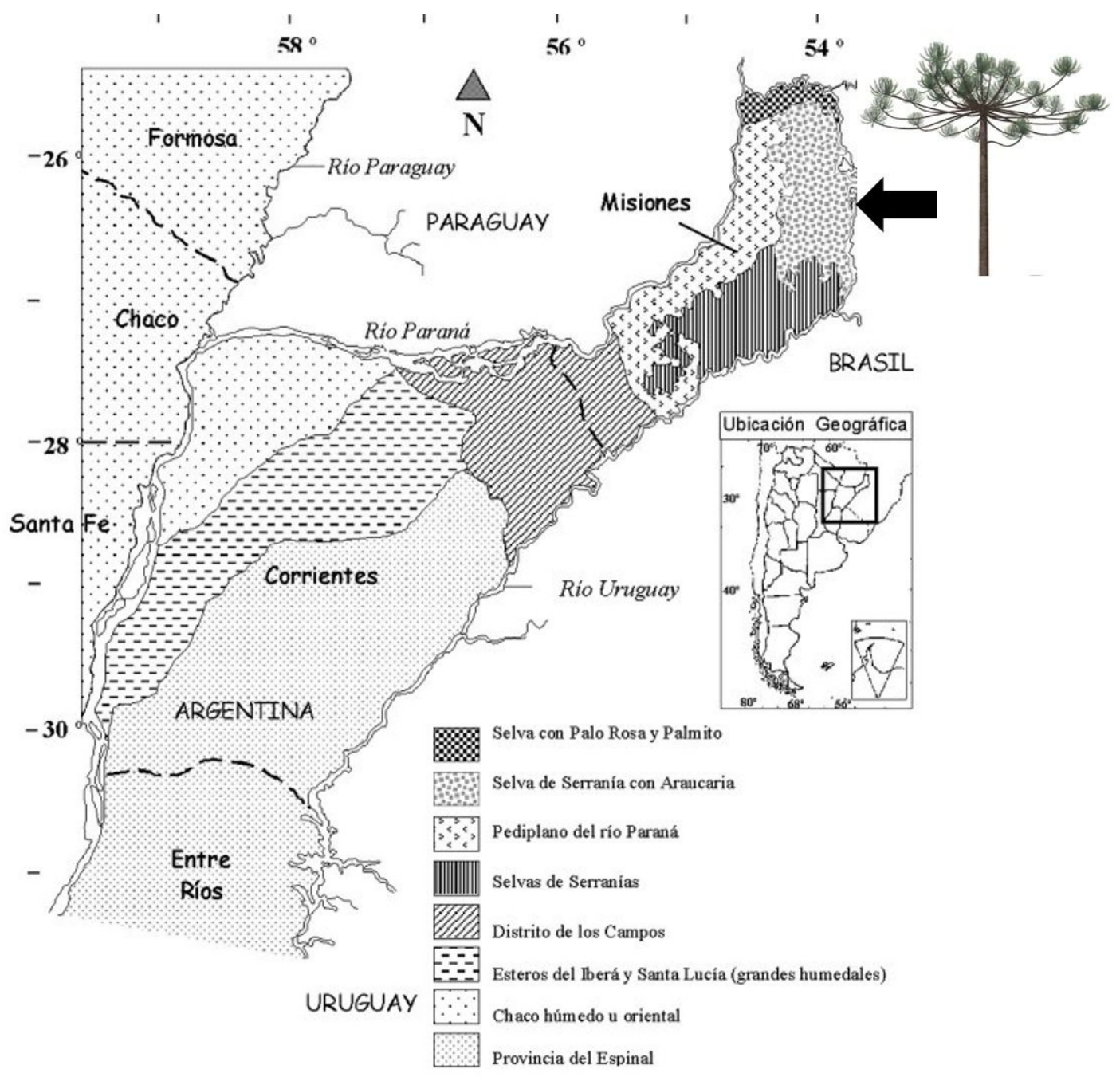

Figura 1. Distribución de Araucaria angustifolia (modificado de Giraudo, 2001). La flecha indica ubicación. 
Recursos genéticos disponibles

Debido a que las semillas pierden viabilidad por deshidratación, alcanzando valores por debajo del 38\% (Eira et al., 1994), la conservación de simientes por períodos de tiempo prolongado representa un gran desafío (Santos et al., 2013). Experimentalmente se conservan semillas de $A$. angustifolia a $0{ }^{\circ} \mathrm{C}$ empaquetadas con películas plásticas manteniendo el poder germinativo durante 9 (Chaves et al., 1999) y 24 meses (Piriz Carrillo et al., 2001; 2003), pero no hay registros de un banco de germoplasma que incluya a la especie en cuestión en su colección. En cuanto a rodales semilleros, en 1948 y mediante un decreto del Programa Estratégico Nacional, se creó la "Reserva de Plantación de Araucaria" posteriormente denominada Estación Forestal Manuel Belgrano, ubicada en San Antonio, Misiones.

La conveniencia del empleo de materiales locales ante el uso de semillas provenientes de Brasil lleva a la conversión de la reserva en una área productora de semillas (Fassola, 2005). Unas 450 ha del Campo Anexo Manuel Belgrano (CAMB) corresponden a plantaciones de Araucaria angustifolia, con edades promedio de 50 años provenientes de distintos orígenes, algunos de ellos de bosques que en la actualidad han desaparecido; por ello se trata de un macizo de características únicas en el país. Además, 1050 ha corresponden a bosques nativos destinados a conservación e investigación (INTA, 2014b).

Los resultados obtenidos han servido para redireccionar el lento pero continuo proceso de transformación de las plantaciones de $A$. angustifolia del CAMB en rodales semilleros en el marco del mejoramiento genético, como también han sentado las bases técnicas para el manejo adaptativo - a nivel paisaje - del área donde permanecen fragmentos del bosque original con predominio de esta especie (Fassola, 2005).

Por otra parte, el Parque Provincial Cruce Caballero -ubicado en el Departamento de San Pedro, Misiones- cuenta con 434.45 ha (Boletín Oficial, 1991). Esta área (el departamento de San Pedro) es la más rica en rodales de araucarias y en 1975 fue declarada por decreto reserva semillera. En 1992 el ME y RNR se encargó de la custodia y cosecha de semillas de araucaria. Esto acompañó al Plan Silvícola Provincial 1993-2000 que promovió la producción en viveros y plantaciones de pino paraná (Burkart, 1993). 
En 1997, un acuerdo entre el Ministerio de Ecología y Recursos Naturales Renovables de Misiones (ME y RNR), la Facultad de Ciencias Forestales de Eldorado y el Centro de Investigaciones y Experiencias Forestales (CIEF), forjó un proyecto de conservación de germoplasma "in situ" y "ex situ" de $A$. angustifolia.

Este acuerdo también pretendió garantizar la provisión de semillas de calidad genética para las forestaciones comerciales de la provincia y crear áreas productoras de semillas en los parques provinciales y en las propiedades del ME y RNR y de la Facultad. Los plantines destinados a plantaciones comerciales han sido exitosamente plantados en sus respectivas propiedades y, de mayo a junio de 1999, la Facultad de Ciencias Forestales entregó al ME y RNR 100 individuos de araucaria de semillas colectadas en el Parque Cruce Caballero, para ser plantadas en áreas degradadas dentro de éste (Bertolini, 1999).

\section{Regeneración de bosques de pino Paraná}

En la década de los 70's el informe sobre el estado de los recursos genéticos forestales de la FAO indicaba que la falta de regeneración de A. angustifolia obedecía en gran parte a la acción del hombre que comercializaba las semillas y practicaba la cría de animales que impedían la regeneración del monte al consumir las semillas nuevas del suelo; la historia resultaba diferente en áreas protegidas donde la intervención antrópica no resultaba dañina confirmándose así que la causa de la falta de regeneración en las masas naturales era la influencia humana (Pitcher, 1973). A esta situación se le suma la predación por parte de la fauna silvestre (Lorenzi, 1992).

Por su escasa viabilidad las semillas germinan tan pronto como caen de la planta madre (Ntima, 1968). A pesar de su temperamento sombrivago la ausencia de plántulas en ambientes sombreados, puede deberse a que es una especie pionera que no se regenera bajo sotobosque (Bittencourt et al., 2004).

El conocimiento de las exigencias ecológicas de la especie permite establecer que la regeneración natural ocurre bajo sombra pero es más eficiente con luz donde se logran mayores crecimientos; en las masas la mayor parte de la regeneración se manifiesta en grupos, en claros resultantes de la corta de árboles. El pastoreo del ganado si bien no daña los renovales si lo hace a las plantas de mayor altura ya que ramonean la corteza. Otra causa de muerte es por el fuego, pero los individuos de 40 o más cm. de diámetro son muy resistentes al mismo (Rogers, 1954). 
Al mismo tiempo, este autor sostiene que la regeneración artificial es una vía factible para la repoblación de la especie. Se ha aceptado generalmente que el método más barato de crear nuevas plantaciones es por siembra directa en lugares que previamente han sido quemados o roturados (Ottone, 2005). Se plantan entre 15.00030.000 semillas/ha y luego se seleccionan las plantas más desarrolladas en el campo. En los cuidados posteriores debe considerarse que los piñones recién germinados pueden ser atacados por animales salvajes (aves y roedores), sobre todo si la oferta de estas semillas es escasa en el campo (IPEF, 2003).

La formación de bosques por plantación resulta una mejor opción si se cuentan con plantines de buena calidad sanitaria y rustificados (Rogers, 1954). Las densidades más frecuentes son de 800 a 1600 plantas/ha con un número de intervenciones consideradas (podas y raleos) según el objetivo de la plantación (Di Marco, 2012).

\section{CONCLUSIONES}

La características ecológicas de Araucaria angustifolia determinan su distribución y por ende su susceptibilidad al cambio climático global. En el contexto de este trabajo ha quedado expuesto como ha sido explotada irracionalmente por su madera, y la expansión agrícola también ha reducido la superficie ocupada por estos bosques. Pese a que hoy en día es una especie protegida y la cosecha de su madera en Brasil está prohibida por la ley, las áreas de distribución natural están fragmentadas y son dispersas, y las grandes poblaciones remanentes son escasas. La situación de conservación de este tipo de bosque es considerada en peligro crítico de extinción. Además, la distribución etaria de las poblaciones remanentes está sesgada hacia las clases más viejas.

En la medida que se tome conocimiento de la importancia que tiene esta especie en las comunidades que la circundan, resulta necesario pensar en un uso del recurso que esté en equilibrio con la conservación de las poblaciones naturales actuales. Al mismo tiempo debe apelarse a todas las herramientas disponibles para frenar la degradación y recuperar áreas sometidas al aprovechamiento desmedido.

Conservar la variación genética es indispensable para implantar estrategias más eficientes de conservación, ya sean éstas in situo ex situ, si se fundan en el conocimiento de los requisitos ambientales y en las pautas de variabilidad de la 
especie contemplando los cambios climáticos futuros. Los programas de conservación de Araucaria podrían verse robustecidos si se realizan programas de seguimiento específicos e investigaciones más dinámicas de su eco fisiología.

\section{BIBLIOGRAFÍA}

Bertolini, M. (1999). Plan de Manejo del Parque Provincial Cruce Caballero. Recuperado de: www.losquesevan.com/archivos/plan-de-manejo-crucecaballero.pdf. (Último acceso: octubre 16 de 2017).

Bittencourt, J., Higa, A., Mazz, M., Ruas, P., Ruas, C., Caccavari, M., Fassola, H. (2004). Conservación, ordenación y uso sostenible de los recursos genéticos de Araucaria angustifolia en Brasil. En Engels, J. (Ed.). Desafíos de la ordenación de los recursos genéticos silvícolas para contribuir a la subsistencia: Ejemplos de Argentina y Brazil (pp. 145-161). IPGRI, Roma, Italia.

Boletín Oficial. (1991). Ley º2876, Boletín Oficial N8154, Posadas, Misiones.

Braier, G. (2004). Estudio de tendencias y perspectivas del sector forestal en América Latina al año 2020- Argentina. Secretaría de Ambiente y Desarrollo Sustentable de la Nación Argentina, Secretaría de Agricultura, Ganadería, Pesca y Alimentos de la Nación Argentina y Organización de las Naciones Unidas para la Agricultura y la Alimentación. 81 pp.

Brasil. (2008). Portaria №83. Lista oficial de espécies da flora brasileira ameaçadas de extinção.

http://www.mma.gov.br/estruturas/ascom_boletins/_arquivos/83_190920080349 49.pdf. (Último acceso: octubre 16 de 2017).

Burkart, R. (1993). Parque Provincial Cruce Caballero. Plan de Manejo para la Producción Sustentable de Semilla de forestales nativas y la conservación de recursos genéticos. Informe final. CFI-Gobierno de la Provincia de Misiones. Misiones, Argentina.

Chaves, A., Mugridge, A., Fassola, H., Alegranza, D., Fernandez, R. (1999). Conservación refrigerada de semillas de Araucaria angustifolia (bert.) $\mathrm{O}$. Kuntze. Bosque 20(2): 117-124.

Colegio de Ingenieros Forestales de Misiones. (2014). Precios de Productos Forestales al cierre de abril 2014. Recuperado de: 
http://www.coiform.com.ar/coiform_leer.php?ati=197. (Último acceso: octubre 16 de 2017).

Di Marco, E. (2012). Ficha técnica: Araucaria angustifolia (Bert.) O. Kuntze. (familia Araucariaceae). Producción forestal 2(4): 48-49.

Eira M., Salomao, A., Dacunha, R., Carrara, D., Mello, C. (1994). Efeito do teor de agua sobre a germinação de sementes de Araucaria angustifolia (Bert.) $\mathrm{O}$. Kuntze-Araucariaceae. Revista brasileira de sementes 16(1): 71-75.

FAO - Food and Agriculture Organization. (1973). Información sobre recursos genéticos forestales $\mathrm{N}^{\circ} 2$. FAO, Roma, Italia.

Fassola, H. (2005). Subprograma pinos en región mesopotámica - Araucaria angustifolia. En Norverto, C. (ed.). Mejores árboles para más forestadores: el programa de producción de material de propagación mejorado y el mejoramiento genético en el Proyecto Forestal de Desarrollo, pp. 43-56. Secretaría de Agricultura, Ganadería, Pesca, y Alimentos, Buenos Aires, Argentina.

Farjon, A. (2006). Araucaria angustifolia. En: IUCN 2011. IUCN Red List of Threatened Species. Recuperado de: http://www.iucnredlist.org. (Último acceso: octubre 12 de 2017).

Fundación Azara. (2009). Estrategias de conservación para el bosque de pino paraná (Araucaria angustifolia) del noreste argentino. Recuperado de: http://www.fundacionazara.org.ar/Investigacion/Proyectos/Pino_parana.htm. (Último acceso: octubre 1 de 2017).

Giraudo, A. R. (2001). La diversidad de serpientes de la Selva Paranaense y del Chaco Húmedo: Taxonomía, biogeografia y conservación. Editorial LOLA, Buenos Aires. 281 pp.

Gnaedinger, S., Herbst, R. (2009). Primer registro de maderas gimnospérmicas de la Formación Roca Blanca (Jurásico Inferior), provincia de Santa Cruz, Argentina. Ameghiniana 46(1): 59-71.

Guillot Ortiz, D., Sanz, G., Rosselló, J. (2006). Claves para el género Araucaria Juss. en la comunidad Valenciana. Bouteloua 1:72-73. 
Herbst, R., Brea, M., Crisafulli, A., Gnaedinger, S., Lutz, A., Martínez, L. (2007). La Paleoxilología en la Argentina. Historia y desarrollo. Ameghiniana 50ำ aniversario (publicación especial) 11: 57-71.

Haene, E.,Aparicio, G. (2014). 100 árboles argentinos. $1^{\circ}$ ed. $4^{\circ}$ reimp. Buenos Aires: Albatros, 128p.

INTA - Instituto Nacional de Tecnología Agropecuaria. (2014) (a). Araucaria, el árbol insignia de Misiones es también una importante alternativa productiva. Recuperado de: http://inta.gob.ar/noticias/araucaria-el-arbol-insignia-demisiones-es-tambien-una-importante-alternativa-productiva. (Último acceso: octubre 11 de 2017).

INTA - Instituto Nacional de Tecnología Agropecuaria. (2014) (b). Campo Anexo Manuel Belgrano. Recuperado de: http://inta.gob.ar/unidades/422006. (Último acceso: octubre 11 de 2017).

IPEF - Instituto de Pesquisas e Estudos Florestais. (2003). Recuperado de: http://www.ipef.br/identificacao/araucaria angustifolia.asp. (Último acceso: octubre 4 de 2017).

Laharrague, P. (2003). Araucaria angustifolia. Species description in the Tropical Tree Seed Manual. Recuperado de: http://www.rngr.net/Publications/ttsm/Folder.2003-07-11.4726. (Último acceso: octubre 2 de 2017).

Lorenzi, H. (1992). Árvores brasileiras: manual de identificação e cultivo de plantas arbóreas nativas do Brasil. 2 v. Nova Odessa: Plantarum, Brasil. 351 p.

Meffe, K., Carroll, R. (1994). Principles of conservation biology. Sinauer Associates, Inc., Sunderland, Massachusetts, USA.

Ntima, O. (1968). The Araucarias. Fast growing timber trees of the lowland tropics $\mathrm{N}^{\circ}$ 3. Department of Forestry. University of Oxford, Oxford.

Ottone, J.R. (2005). "Arboles forestales”. Ed. AgroVet S.A. Buenos Aires. 571 pp.

Piriz Carrillo, V., Fassola, H., Chaves, A., Mugridge, A. (2001). Influencia de la temperatura y composición de la atmósfera en la conservación de la capacidad germinativa de semillas de Araucaria angustifolia (Bert.) O. Kuntze almacenadas por un período prolongado. Revista forestal latinoamericana 16(30): 163-178. 
Piriz Carrillo, V., Fassola, H., Chaves, A., Mugridge, A. (2003). Refrigerated storage of seeds of Araucaria angustifolia (Bert.) O. Kuntze over a period of 24 months. Seedscience and technology 31(2): 411-421.

Pitcher, J. (1973). Recursos genéticos de Araucaria angustifolia (Bert.) O. Ktze, en el Brasil. Recuperado de: http://www.fao.org/docrep/006/e4209s/e4209s10.htm. (Último acceso: septiembre 26 de 2017).

Pujana, R., Umazano, A.,Bellosi, E. (2007). Maderas fósiles afines a Araucariaceae de la Formación Bajo Barreal, Cretácico tardío de Patagonia central (Argentina). Revista del museo argentino de ciencias naturales 9(2): 161-167.

Rodríguez, M., Cardozo, A., Ruiz Díaz, M., Prado, D. (2005). Los bosques nativos misioneros: estado actual de su conocimiento y perspectivas. En Arturi, M., Frangi J. y Goya J. (eds.). Ecología y manejo de los bosques de Argentina, pp. 3-33. La Plata, Argentina: EDULP.

Rogers, L. 1954. El cultivo del pino Paraná. Unasylva 8(11): 15-18.

Salazar, R., Soihet, C., Méndez, J. (2000). Manejo de semillas de 100 especies forestales de América Latina. CATIE, Proyecto de Semillas forestales: Danida Forest Seed Centre, Turrialba, Costa Rica. 204p.

Sebbenn, A., Almeida Saraiva Pontinha, A., Giannetti, E., Kageyama, P. (2003). Conservación ex situ de Araucaria angustifolia (bert.) O. Ktze. en el estado de São Paulo, Brasil. Recuperado de: http://www.fao.org/docrep/005/y4341s/Y4341S05.htm. (Último acceso: octubre 9 de 2017).

Silveira Wrege, M., Higa, R., Miranda Britez, R., Cordeiro Garrastazu, M., De Sousa, V., Caramori, P., Radin, B., Braga, H. (2009). El cambio climático y la conservación de Araucaria angustifolia en Brasil. Unasylva 60: 231-232.

Véliz Pérez, M., Barrios, A., Dávila Pérez, C. (2007). Actualización Taxonómica de la Flora de Guatemala, Capítulo 1. Pinophyta (Coníferas) - informe final. Herbario BIGU, Escuela de Biología, FCQF, DIGI, USAC, Guatemala.

Waters, T. (2003). Systematics of Agathis Salisb. Recuperado de: http://www.agathis.info/index.php. (Último acceso: septiembre 23 de 2017). 
Wendling, I., Dutra, L., Hoffmann, H., Bettio, G., Hansel, F. (2009). Indução de brotações epicórmicas ortotrópicas para a propagação vegetativa de árvores adultas de Araucaria angustifolia. Agronomía Costarricense 33(2): 309-319. 